\title{
Gallbladder Neuroendocrine Tumors: Is There a Need for a Specific Approach?
}

\author{
Vijay Waman Dhakre ${ }^{a}$ Govind Purushothaman ${ }^{b}$ Nilesh Doctor ${ }^{b}$ \\ aDepartment of Surgery, Lokmanya Tilak Municipal Medical College and General Hospital, Mumbai, India; \\ ${ }^{b}$ Department of Surgical Gastroenterology \& HPB Surgery, Jaslok Hospital and Research Centre, Mumbai, India
}

\section{Keywords}

Gallbladder neuroendocrine tumor · Small cell neuroendocrine carcinoma $\cdot$ Gastrointestinal neuroendocrine tumors

\section{Abstract}

Neuroendocrine tumors (NETs) of the gallbladder or the biliary tree are rare. Most of the current guidelines and protocols are derived from the experience of managing lung small cell neuroendocrine carcinoma or gastrointestinal NETs. But, the overall outcome of gallbladder NETs (GB-NETs) seems worse than similarly staged lung NETs and adenocarcinoma of the gallbladder. This may be due to its rarity and lack of literature for a focused approach toward its treatment. Hence, the need for a specifically designed approach might help improve results of treatment for these rare tumors. We share our experience of 2 patients with GB-NETs and their 5 -year outcome.

(C) 2021 The Author(s) Published by S. Karger AG, Basel

karger@karger.com www.karger.com/gat

Karger"

GOPEN ACCESS
(C) 2021 The Author(s).

Published by S. Karger AG, Basel

This is an Open Access article licensed under the Creative Commons Attribution-NonCommercial-4.0 International License (CC BY-NC) (http://www.karger.com/Services/OpenAccessLicense), applicable to the online version of the article only. Usage and distribution for commercial purposes requires written permission.

\section{Introduction}

As per the WHO classification of NETs, G3 is now further subdivided into well- to poorly differentiated neuroendocrine carcinoma. The poorly differentiated variety could be of a small cell (SCNEC) or large cell variety. SCNEC of the GI tract is rare - around $0.1-1 \%$ of all malignancies and even rarer in the biliary tract [1]. Due to its low incidence, treatment guidelines rely on recommendations coming from the data and experience from lung SCNEC [2]. This may not be appropriate as prognosis between the two varies widely. The median survival for SCNEC of the GI tract is 6-12 months, which is shorter than that for the lung [3].

Even more so there are very limited data available on the exact pathophysiology of GB-NEC, molecular triggers, specific immunohistochemistry (IHC) and molecular testing, and natural course of the disease related to it [4]. Due to the same reasons, GB-NEC responds poorly to the traditional chemotherapy and radiotherapy regimen available [4]. Hence, more detailed insights into its molecular nature and genetics along with high-quality clinical evidence are required to adopt a more appropriate management strategy tailored specially for GB-NEC.

Correspondence to:

Vijay Waman Dhakre, vddrvijayd@gmail.com 


\section{Case Report/Case Presentation}

Case 1

A 58-year-old gentleman presented with right hypochondriac severe nonradiating pain for 2-3 months associated with nausea. He had similar pain 5 years back. It was not associated with fever with chills or jaundice. The pain was not associated with any change after feeding. There was no evidence of weight loss or melena/acholic stools.

Clinically, the patient had a heart rate of 84 beats/min, blood pressure of $138 / 88 \mathrm{~mm} \mathrm{Hg}$, and no evidence of icterus. The radiograph of the abdomen was normal, and an ultrasound of the abdomen showed multiple tiny gallbladder calculi with sludge and normal common bile duct (CBD).

With the diagnosis of symptomatic cholelithiasis, the patient was posted for laparoscopic cholecystectomy. Standard laparoscopic cholecystectomy was done and was eventful. The gallbladder histopathology report was suggestive of poorly differentiated carcinoma with neuroendocrine features with small cell carcinoma; IHC was done which showed diffusely positive for CD56/ NCAM, distinctly positive for AE1/AE3 and EMA, and negative for synaptophysin, smooth muscle actin, and desmin.

At this stage, the patient was referred to our institution. His complete blood count and liver and renal function tests were within normal limits. CA 19-9 was normal (12.7 U/mL).

Fluorodeoxyglucose (FDG)-positron emission tomography (PET) computed tomography (CT) was done which showed a hypermetabolic focus in hepatic parenchyma of the liver adjacent to the gallbladder fossa with a maximum standardized uptake value (SUVmax) of 4.3 and a periportal lymph node with low-grade FDG SUVmax of 1.9. After 8 weeks of cholecystectomy, we planned a completion radical cholecystectomy for him. Portal lymph nodes around the right and left hepatic artery, portal vein, and CBD were excised, and the gallbladder fossa (segments $4 \mathrm{~B}$ and V) was excised (Fig. 1).

Histopathology and IHC of this specimen suggested that it is a poorly differentiated small cell carcinoma of biliary origin with neuroendocrine positive markers like synaptophysin and CK7, and resected surgical margins were negative. As per WHO grading, it was a SCNEC with poor differentiation and a high grade (Fig. 2).

The patient was followed up in the next 3 months and 6 months with transabdominal sonography which was normal. After 6 months, the patient was lost to follow-up. The patient presented after 30 months of extended cholecystectomy with abdominal distension, weight loss, and jaundice. Transabdominal ultrasound was suggestive of multiple liver metastases with para-caval and para-aortic lymphadenopathy with gross ascites; multiple omental and mesenteric nodules were seen. The patient died after 31 months of radical cholecystectomy.

\section{Case 2}

A 67-year-old gentleman presented with right hypochondriac pain which was colicky and referred to the ipsilateral back for 2 days. It was associated with excessive bloating, nausea, and abdominal discomfort. There was no history of fever with chills, jaundice, loss of appetite or weight loss, or retrosternal burning pain sensation. He was diagnosed with diabetes mellitus and was on oral antihypoglycemic which was well controlled.

His physical examination was normal, and the abdomen was soft without any evidence of tenderness or any lump. There was no evidence of ascites or icterus.

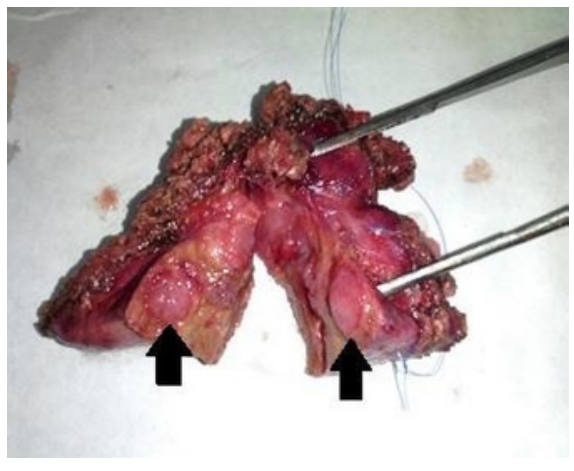

Fig. 1. Case 1. Postoperative cut-opened specimen of the liver from extended cholecystectomy showing nodular lesions (black arrows) showing liver metastases from the primary small cell carcinoma neuroendocrine carcinoma of the gallbladder.

His complete blood count and liver and renal function tests were normal. His transabdominal ultrasound showed a mobile gallstone of $1 \mathrm{~cm}$ and a normal CBD.

Standard laparoscopic cholecystectomy was performed in the same admission, and the gallbladder specimen showed cord and nest tumor cells infiltrating beyond the muscularis mucosa up to subserosal fat. IHC stained positive for synaptophysin, chromogranin, and an MIB-1 labeling index of 1-2\%. As per WHO grading, it was labeled as NET, G1 (Fig. 3). The patient is clinically stable and is not having lesions or recurrence 5 years after the cholecystectomy as evident from follow-up with USG and MRCP.

\section{Discussion/Conclusion}

GB-NETs are rare lesions of the gallbladder accounting for $0.5-1 \%$ of all NETs $[5,6]$. SCNEC is even rarer (0.1-0.4\% of all extrapulmonary SCNECs) and more aggressive [6-8]. SCNEC is common in pulmonary tissue, but it can arise from extrapulmonary sites like the esophagus, stomach, colon, rectum, gallbladder, salivary gland, cervix, urinary bladder, prostate, skin, and unknown primary site with metastases [9]. In the gallbladder, it comprises $1-5 \%$ of all gallbladder malignancies, shows a propensity toward females in their sixth decades, and 60$66 \%$ have incidental or presenting features suggesting cholelithiasis $[6,9,10]$.

GB-NET has many theories regarding its pathogenesis; a general assumption is that there are no neuroendocrine cells in the gallbladder. It is shown that neuroendocrine cells do exist in gallbladders of patients who have cholelithiasis; many believe that the epithelium develops metaplastic changes in the presence of calculi or inflammation which develops into neuroendocrine cells. Others believe that these cells develop from the gallbladder adenocarci- 
Fig. 2. Case 1. Small cell neuroendocrine tumor. a The tumor is composed of poorly differentiated oval to polygonal cells with hyperchromatic nuclei (H\&E stain. $\times 100)$. b Tumor cells show pleomorphic nuclei with mitotic activity (H\&E. ×400). c CK7 positivity in the tumor cells. d Synaptophysin positivity in the tumor cells. e CK7 positivity in the metastatic carcinoma in the lymph node. $f$ MIB-1 proliferation in the tumor cells.
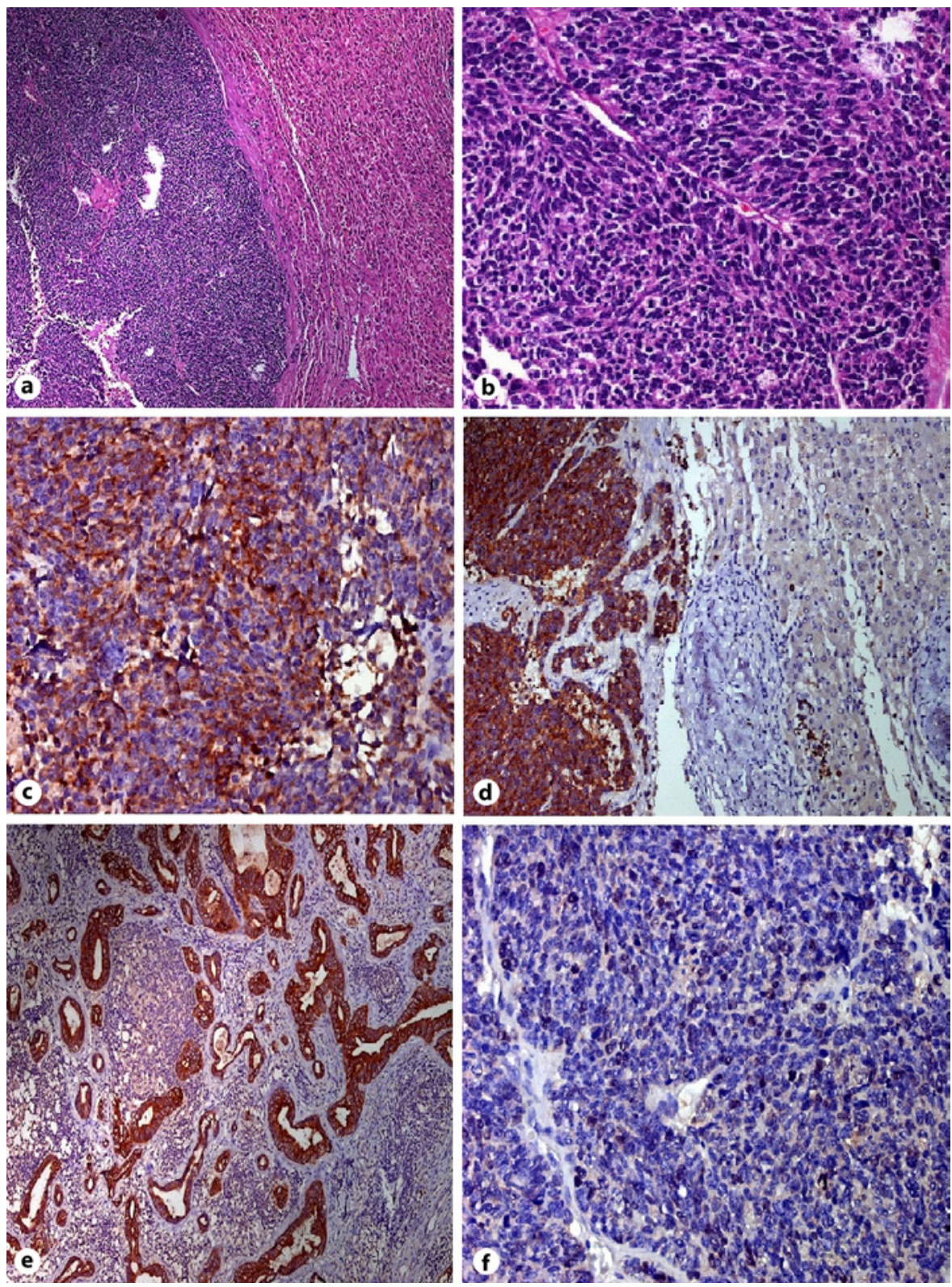

noma or a totipotent stem cell and grow with complimenting one another giving rise to the so-called mixed neuro nonneuroendocrine neoplasm which was earlier (in 2010) called mixed adenoneuroendocrine carcinomas $[11,12]$.

The WHO 2019 and the International Agency for Research in Cancer (IARC) 2017 has basically classified these tumors into well-differentiated NETs and poorly differentiated NECs. A well-differentiated lesion (NET) can be a G1, G2, or G3 lesion. The G3 lesion can be well differentiated or poorly differentiated, but is always a high-grade lesion. The low, intermediate, or high grade is based on mitotic rate and Ki-67 index: $<2,2-20,>20$ and $<3,3-20$, and $>20$, respectively. Poorly differentiated G3 lesions are small cell or large cell NECs which have a high mitotic rate and a Ki-67 index of $>20$ [12]. Also, they can be distinguished into NET and NEC based on the molecular signature they show like mutations seen in MEN1, $D A X X$, and ATRX are well-differentiated NETs, whereas mutations in TP53 and/or RB1 are said to be poorly differentiated NECs. Tumors showing $(\geq 30 \%)$ each component in the histopathological analysis of both adenocarcinoma and NETs are classified separately [12]. 

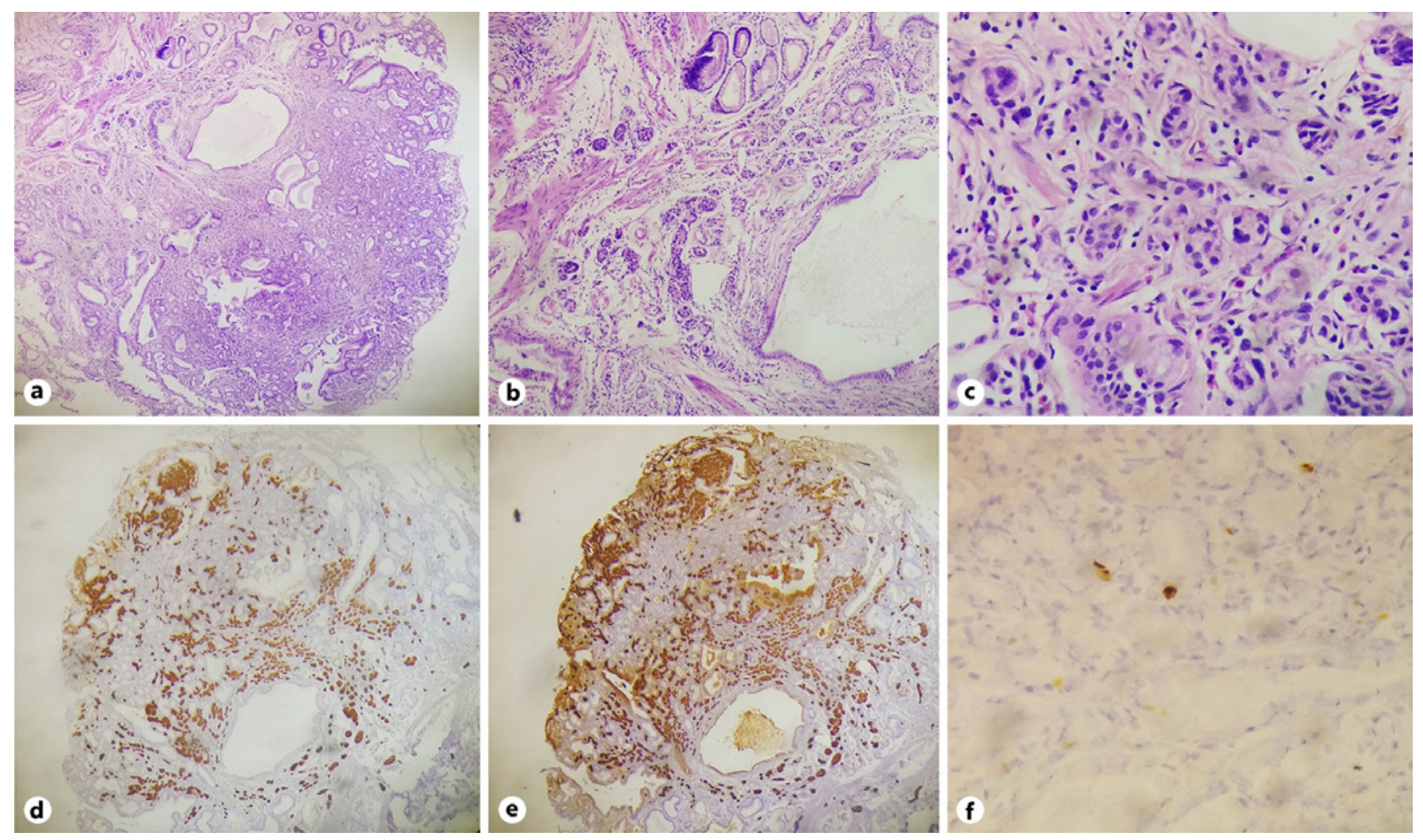

Fig. 3. Case 2. a Gallbladder NET polyp. b Tumor infiltrating the muscularis mucosa. c Tumor cells in cords and nests in high power. d Positive immunohistochemistry for synaptophysin in low power. e Positive immunohistochemistry for chromogranin in low power. $\mathbf{f}$ MIB-1 labeling index $1-2 \%$ in high power.

Clinically, patients present with right upper quadrant discomfort to a pain similar to cholelithiasis. No specific signs or symptoms pertaining to the hormones or peptides secreted by the smaller GB-NETs are seen mainly because of extensive first-pass metabolism by the liver and deactivation of these peptides unlike NET of other organs where before getting deactivated, it shows a typical clinical syndrome which can aid in its diagnosis. But, huge GB-NET masses that are active can produce diarrhea $[7,11,13]$. Mostly these are identified incidentally on histopathological examination after cholecystectomy.

Routine imaging studies that are done for evaluating patients like transabdominal ultrasonography, contrastenhanced CT, and MRI/MRCP will retrospectively show gallstones, a polyp, or a mass with/without enhancement, but no specific diagnostic signs for GB-NETs are seen [7, 14]. Some studies have analyzed MRI features and had some features which can help differentiate GB-NET from gallbladder adenocarcinoma: the GB-NET will have a thicker rim appearance on contrast-enhanced MRI and in diffusion-weighted images, and if we consider the ratio of apparent diffusion values between the spleen and the lesion (primary/metastatic/lymph node), the ratio will be lower in GB-NET compared to gallbladder adenocarcinoma [15]. FDG-PET/CT is used rather than gallium-68 DOTATOC as the former is more positive in these cases than the latter (92\% vs. 50\%) [16].

A confirmatory diagnosis of suspicious lesions will be done on a biopsy which on IHC will show specific markers for neuroendocrine origins like synaptophysin and chromogranin-A; grading (Ki-67 mitotic rate) and differentiation must be obtained $[11,15,16]$. SCNEC shows small- to medium-sized oval- to round-shaped cells on histopathology, with hyperchromatic nuclei along with indistinct nucleoli arranged in sheets and nests $[17,18]$.

The usual approach is to stage these lesions based on their TNM staging. Lesions that are operable and can be safely resected should undergo surgical resection, while those that are disseminated should be subjected to sys- 
temic chemotherapy based on platinum chemotherapeutic agents. Early detection of the lesion and its aggressive surgical management with $\mathrm{R} 0$ resection has resulted in prolonged survival. But, there are no direct studies that compare curative resection versus chemotherapy versus concomitant chemoradiotherapy [5].

SCNEC of the gallbladder has been traditionally treated similar to small cell lung neoplasm in terms of platinum-based systemic/intra-arterial chemotherapeutic agents due to fact that it shared similar biologic behavior, clinical course, and showed a similar response to treatment as seen in the small cell lung cancers $[10,19]$. But, GB-SCNEC is different from the lung counterpart in terms of its etiology (smoking is common in the lung); lung small cell carcinoma has a higher frequency of brain metastasis and shows a better response to platinum chemotherapy than the GB-SCNEC $[9,19]$. GB-SCNEC is also different in other areas like it shows lower rates of mutations in the TP53 gene and less altered RB1 gene. Initially, it was thought that the GIT NEC behaves similarly and should be looked upon as one, but we should not believe that all NECs of biliary, pancreatic, or GI origin are one and treat them equally; instead, we should consider developing a dedicated management algorithm based on clinical behavior, tumor origin, location, and biology (e.g., GB-SCNEC) [3, 20].

Poorly and well differentiated are distinct in terms of outcome. Carcinoid tumors of GB had a median survival of 11 months, GB-NEC G3 poorly differentiated was 9.8 months, SCNEC was 4 months, and GB adenocarcinoma was 10.3 months. If we have to consider carcinoid and SCNEC 1-year and 5-year survival, it is $62 \%$ and $37 \%$ for carcinoid and $21 \%$ and $0 \%$ for SCNEC, respectively [21]. This holds true for the disease-free survival too; the median disease-free survival for G1-G2 NET was 55 months and 11 months in poorly differentiated NEC [22].

The overall median survival rate for G3-NET poorly differentiated variety is $4-13$ months, 1 -year survival is $21 \%$, and nearly 0 beyond 5 years $[7,8]$. SCNECs are more aggressive tumors, and more than half of the patients have an inoperable disease on presentation, and only around $25 \%$ will be within operable stages [23]. In the study done by Soga et al. [24], they found that $32.4 \%$ of poorly differentiated NECs were operable, and $77.2 \%$ in the carcinoid group was in an operable stage with curative intent. As per Surveillance, Epidemiology, and End Results data, none of the SCNEC patients are alive after 10 years versus $36 \% 10$-year survival in the case of carcinoid tumors $[7,13]$. Even the overall survival scores for GBNEC are worse than similarly staged gallbladder adeno-

Gallbladder Neuroendocrine Tumor carcinomas [25]. GB-NEC versus GB adenocarcinoma after matching the propensity score showed that GBNEC had worse prognosis compared to GB adenocarcinoma (3-year survival of 31.1\%-63.8\%) [25].

Higher performance scores, Ki- 67 of $<55 \%$, nonelevated lactate dehydrogenase ( $<2$ times the upper normal limit), and non-hepato-pancreato-biliary site of origin were associated with better survival [20,26, 27]. Traditionally, platinum-based chemotherapy was used, but many studies are using irinotecan and etoposide combined with cisplatin which has shown better efficacy but lacks randomized trials $[20,26,27]$. But, the platinum-based regimen has shown a response rate of around $50 \%$, much less than that in lung SCNEC; this may be because in GIT, there may be a much higher mix of histological variants that may plunge the response even down [3].

A combination treatment approach like adjuvant platinum-based therapy in selected cases coupled with R0 resection is the current best possible management. But, the distant spread will need only systemic chemotherapy. This management is based on the lung SCNEC experience, but as shown in gallbladder adenocarcinoma, aggressive surgical management has marginally improved survival. SCNEC or poorly differentiated G3-NET may also benefit from a similar aggressive approach [4]. Rarity of incidence, lack of data regarding its genetic and molecular signatures to guide therapy, and paucity in the literature may be the reasons behind the high recurrence rate, inadequate response to conventional therapy, and associated mortality [4].

NET is staged as per TNM staging, and separate staging system is in place for various tissues of origin. Treatment of well-differentiated grade 3 NET is based on favorable biology, which is when the Ki-67 index is $<55 \%$ and may have a positive somatostatin receptor (SSR)based PET imaging (SSR-PET) which indicates that the tumor is slow growing. Ki-67 $>55 \%$ with a negative SSRPET may have an unfavorable biology. Overall management is as follows [28].

Resection is done in favorable lesions. Trail of neoadjuvants is used for locally advanced or metastatic lesions which is found to have an unfavorable biology. For favorable biology with locally advanced or metastatic lesions, resections can be attempted whenever possible. In cases where patients have metastatic disease but are asymptomatic and have lower tumor burden, close follow-up with imaging is used (12-24 weekly imaging for the first 2 years). In cases of positive hormonal signs and symptoms with SSR-PET positive, octreotide/lanreotide is used. Peptide receptor radionuclide therapy with $177 \mathrm{Lu}-$ dotatate can be used in selected patients [28]. 
Treatment options of poorly differentiated NET (including SCNEC and LCNEC) are neoadjuvant chemotherapy, surgical resection, and adjuvant chemotherapy with or without radiotherapy/radiotherapy as a solo treatment. Treatment is tailored considering anatomical location. For unresectable tumors, only chemotherapy or chemotherapy with concurrent chemoradiotherapy is planned. In metastatic cases, chemotherapy is alone planned [28].

To summarize, we have described 2 cases from the wide spectrum of differentiation of GB-NETs with G1NET and SCNEC, out of which SCNEC is aggressive and may need belligerent management, while G1-NET is commonly encountered incidentally (i.e., after cholecystectomy). Cholecystectomy suffices in G1-NET of GB with Tis and T1 lesion, with close follow-ups. The natural course of the NEC will depend majorly on the Ki-67 index, well or poorly differentiated histology, and able to achieve an R0 resection [27, 28]. Due to its rarity and very fewer cases described in the literature, we are not able to currently formulate a more specific protocol to achieve a better outcome; the same can be achieved by studying a larger number of patients and amalgamating pooled data from randomized controlled trials.

\section{Statement of Ethics}

Written informed consent was obtained from the patients for publication of this case report and any accompanying images. Also, consent was obtained to publish photos and hide identity.
This case was reviewed by the Director of Surgical Gastroenterology, and it was determined that since it is a case report, it does not require any further approvals.

\section{Conflict of Interest Statement}

The authors have no conflicts of interest to declare.

\section{Funding Sources}

There are no funding sources to declare.

\section{Author Contributions}

As per ICMJE Criteria for Authorship (http://www.icmje.org/ recommendations/browse/roles-and-responsibilities/definingthe-role-of-authors-and-contributors.html), Vijay W. Dhakre designed the article and also contributed to conception and literature search. Govind Purushothaman contributed to analysis and literature search and helped procure case details and photographs. Nilesh Doctor contributed to literature search, discussion analysis, and final proofreading.

\section{Data Availability Statement}

All data generated or analyzed during this study are included in this article. Further enquiries can be directed to the corresponding author.

\section{References}

1 Miyashita T, Konishi K, Noto M, Taniguchi K, Kaji M, Kimura H, et al. Nihon Shokakibyo Gakkai Zasshi. 2001;98(10):1195-8.

2 Brenner B, Tang LH, Shia J, Klimstra DS, Kelsen DP. Small cell carcinomas of the gastrointestinal tract: clinicopathological features and treatment approach. Semin Oncol. 2007;34(1):43-50.

3 Brenner B, Shah MA, Gonen M, Klimstra DS, Shia J, Kelsen DP. Small-cell carcinoma of the gastrointestinal tract: a retrospective study of 64 cases. Br J Cancer. 2004;90(9):1720-6.

4 Liu F, Li Y, Ying D, Qiu S, He Y, Li M, et al. Whole-exome mutational landscape of neuroendocrine carcinomas of the gallbladder. Signal Transduct Target Ther. 2021;6(1):55.

5 You YH, Choi DW, Heo JS, Han IW, Choi SH, Jang KT, et al. Can surgical treatment be justified for neuroendocrine carcinoma of the gallbladder? Medicine. 2019;98(11):e14886.
6 Moris D, Tsilimigras DI, Lim J, Camastra D, Nanavati A, Knechtle SJ, et al. Neuroendocrine carcinomas of the gallbladder: lessons learnt from cases at opposite ends of the spectrum. J BUON. 2018;23(6):1922-6.

7 Adachi T, Haraguchi M, Irie J, Yoshimoto T, Uehara R, Ito S, et al. Gallbladder small cell carcinoma: a case report and literature review. Surg Case Rep. 2016;2(1):71.

8 Carrera C, Kunk P, Rahma O. Small cell carcinoma of the gallbladder: case report and comprehensive analysis of published cases. J Oncol. 2015;2015:304909.

9 Brennan SM, Gregory DL, Stillie A, Herschtal A, Mac Manus M, Ball DL. Should extrapulmonary small cell cancer be managed like small cell lung cancer? Cancer. 2010;116(4):888-95.

10 Fujii $\mathrm{H}$, Aotake T, Horiuchi T, Chiba Y, Imamura Y, Tanaka K. Small cell carcinoma of the gallbladder: a case report and review of 53 cases in the literature. Hepatogastroenterology. 2001;48(42):1588-93.
11 Liu W, Chen W, Chen J, Hong T, Li B, Qu Q, et al. Neuroendocrine carcinoma of gallbladder: a case series and literature review. Eur J Med Res. 2019;24:8.

12 Nagtegaal ID, Odze RD, Klimstra D, Paradis V, Rugge M, Schirmacher P, et al. WHO classification of tumours of the digestive system. Histopathology. 2020 Jan;76(2):182-8.

13 Li M, Liu F, Zhang Y, Wu X, Wu W, Wang $\mathrm{XA}$, et al. Whole-genome sequencing reveals the mutational landscape of metastatic smallcell gallbladder neuroendocrine carcinoma (GB-SCNEC). Cancer Lett. 2017;391:20-7.

14 Niu C, Wang S, Guan Q, Ren X, Ji B, Liu Y. Neuroendocrine tumors of the gallbladder (Review). Oncology Letters. 2020;19:3381-8.

15 Bae JS, Kim SH, Yoo J, Kim H, Han JK. Differential and prognostic MRI features of gallbladder neuroendocrine tumors and adenocarcinomas. Eur Radiol. 2020;30(5):2890901 
16 Walter T, Tougeron D, Baudin E, Le Malicot $\mathrm{K}$, Lecomte T, Malka D, et al. Poorly differentiated gastro-entero-pancreatic neuroendocrine carcinomas: are they really heterogeneous? Insights from the FFCD-GTE national cohort. Eur J Cancer. 2017;79:158.

17 Sciarra A, Missiaglia E, Trimech M, Melloul E, Brouland JP, Sempoux C, et al. Gallbladder Mixed Neuroendocrine-Non-neuroendocrine Neoplasm (MiNEN) arising in intracholecystic papillary neoplasm: clinicopathologic and molecular analysis of a case and review of the literature. Endocr Pathol. 2020; 31(1):84-93.

18 La Rosa S, Sessa F. High-grade poorly differentiated neuroendocrine carcinomas of the gastroenteropancreatic system: from morphology to proliferation and back. Endocr Pathol. 2014;25(2):193-8.

19 Walenkamp AM, Sonke GS, Sleijfer DT. Clinical and therapeutic aspects of extrapulmonary small cell carcinoma. Cancer Treat Rev. 2009;35(3):228-36.
20 Sorbye H, Welin S, Langer SW, Vestermark LW, Holt N, Osterlund P, et al. Predictive and prognostic factors for treatment and survival in 305 patients with advanced gastrointestinal neuroendocrine carcinoma (WHO G3): the NORDIC NEC study. Ann Oncol. 2013;24(1): 152-60.

21 Eltawil KM, Gustafsson BI, Kidd M, Modlin IM. Neuroendocrine tumors of the gallbladder: an evaluation and reassessment of management strategy. J Clin Gastroenterol. 2010; 44(10):687-95.

22 Tang LH, Untch BR, Reidy DL, O'Reilly E, Dhall D, Jih L, et al. Well-differentiated neuroendocrine tumors with a morphologically apparent high-grade component: a pathway distinct from poorly differentiated neuroendocrine carcinomas. Clin Cancer Res. 2016; 22(4):1011-7.

23 Nau P, Liu J, Dillhoff M, Forster M, Hazey J, Melvin S. Two cases of small cell carcinoma of the gallbladder. Case Rep Med. 2010;2010: 453624.

24 Soga J. Carcinoids and their variant endocrinomas. An analysis of 11842 reported cases. J Exp Clin Cancer Res. 2003;22(4):517-30.
25 Yan S, Wang Y, Chen X, Zhang Y, Huang Z, Zhao J, et al. Clinical analysis of 15 cases of gallbladder neuroendocrine carcinoma and comparison with gallbladder adenocarcinoma using a propensity score matching. Cancer Manag Res. 2020;12:1437-46.

26 Yamaguchi T, Machida N, Morizane C, Kasuga $\mathrm{A}$, Takahashi $\mathrm{H}$, Sudo $\mathrm{K}$, et al. Multicenter retrospective analysis of systemic chemotherapy for advanced neuroendocrine carcinoma of the digestive system. Cancer Sci. 2014;105(9):1176-81.

27 Chen H, Shen YY, Ni XZ. Two cases of neuroendocrine carcinoma of the gallbladder. World J Gastroenterol. 2014;20(33):1191620.

28 Shah $\mathrm{MH}$, Goldner WS, Benson AB, Bergsland E, Blaszkowsky LS, Brock P, et al. Neuroendocrine and adrenal tumors, version 2.2021, NCCN Clinical Practice Guidelines in oncology. J Natl Compr Canc Netw. 2021; 19(7):839-68. 